\title{
Papst Die Medien - ein Netzwerk für Benedikt XVI. Kommunikation, Gemeinschaft und Kooperation \\ Botschaft zum 40. Welttag der sozialen Kommunikationsmittel
}

\section{Liebe Brïder und Schwestern!}

1. Es ist mir eine Freude, in zeitlicher Nähe zur 40. Wiederkehr der Abschlusses des II. Vatikanischen Ökumenischen Konzils dessen Dekret über die Sozialen Kommunikationsmittel „Inter Mirifica“ in Erinnerung rufen zu dürfen, das insbesondere die Macht der Medien, die gesamte menschliche Gesellschaft zu beeinflussen, anerkannt hat. Die Notwendigkeit, jene Macht im Interesse der ganzen Menschheit zu zügeln, hat mich veranlasst, in dieser meiner ersten Botschaft zum Welttag der Kommunikationsmittel kurz über die Vorstellung von Medien als einem Netzwerk, das Kommunikation, Gemeinschaft und Kooperation ermöglicht, zu reflektieren.

Der Hl. Paulus beschreibt in seinem Brief an die Epheser lebendig unsere menschliche Berufung, „Anteil an der göttlichen Natur zu haben“ (cf. Dei Verbum, 2): Durch Christus haben wir in einem Geist Zugang zum Vater; daher sind wir nicht länger Fremde und Fremdartige, sondern mit den Heiligen Bürger und Mitglieder im Hause Gottes, die zu einem heiligen Tempel heranwachsen, eine Wohnstatt für Gott (cf. Eph 2, 18-22). Dieses grosse Bild eines Lebens in Gemeinschaft erfasst alle Aspekte unseres Lebens als Christen. Der Aufruf, der Selbstmitteilung Gottes in Christus treu zu sein, ist in der Tat eine Aufforderung, dessen dynamische Kraft in uns zu erkennen, die danach strebt, sich nach aussen gegenüber anderen mitzuteilen, so dass seine Liebe wirklich der vorherrschende Massstab für die Welt werden kann (cf. Predigt beim Weltjugendtag, Köln, 21. August 2005).

2. Technologische Fortschritte im Medienbereich haben in gewisser Hinsicht Zeit und Raum erobert und Kommunikation zwischen Menschen auch im Fall grosser Entfernungen zum selben Zeitpunkt ohne Zeitversetzung unmittelbar möglich gemacht. Diese Entwicklung stellt ein enormes Potential für den Dienst am Gemeinwohl dar und ein „Gut, das geschützt und gefördert werden muss" (cf. Die schnelle Entwicklung, 10). Wie wir alle wissen, ist unsere Welt jedoch bei weitem nicht vollkommen. Täglich werden wir daran erinnert, dass Unmittelbarkeit der Kommunikation nicht notwendig Entwicklung von Zusammenarbeit und Gemeinschaft in der Gesellschaft heisst. 
Die Gewissen der Menschen zu bilden und ihr Denken formen zu helfen ist niemals eine leichte Aufgabe. Echte Kommunikation verlangt auf Prinzipien gestützten Mut und Einsatz. Sie erfordert die Entschiedenheit der Medienschaffenden, nicht unter dem Gewicht der Informationsfülle müde zu werden und sich auch nicht mit partiellen oder provisorischen Wahrheiten zufrieden zu geben. Im Gegenteil ist es notwendig, sich um die letzte Begründung und Bedeutung menschlicher, persönlicher und sozialer Existenz zu bemühen und dies zu verbreiten (cf. Fides et Ratio, 5). Auf diese Weise können die Medien konstruktiv zur Verbreitung all dessen, was gut und wahr ist, beitragen.

3. Der an die Medien von heute gerichtete Aufruf zu verantwortlichem Verhalten - Vorkämpfer der Wahrheit und Förderer des Friedens, der daraus folgt, zu sein - bringt eine Reihe von Herausforderungen mit sich. Die verschiedenen Instrumente sozialer Kommunikation ermöglichen zwar den Austausch von Information, Ideen und gegenseitiges Verstehen, sind aber von Doppeldeutigkeiten betroffen. Neben dem Begriff eines "grossen runden Tisches" zum Dialog verursachen gewisse Tendenzen in den Medien eine Art Monokultur, die kreatives Talent dämpft, die Subtilität komplexen Denkens reduziert und die Besonderheit kultureller Verhaltensweisen und religiösen Glaubens unterbewertet. Dies sind Verzerrungen, die sich ergeben, wenn die Medien-Industrie zum Selbstzweck wird oder nur gewinnorientiert arbeitet und den Sinn für die Verantwortlichkeit gegenüber dem Gemeinwohl verliert.

Weiter bedarf es immer steter Ermutigung zu präziser Berichterstattung über Ereignisse, vollständiger Erläuterung von Sachverhalten und Vorgängen öffentlichen Interesses sowie fairer Darstellung verschiedener Auffassungen und Gesichtspunkte. Von besonderer Wichtigkeit ist es, Ehe und Familienleben hochzuhalten und zu unterstützen, eben weil es zu den Fundamenten jeder Kultur und Gesellschaft gehört (cf. Apostolicam Actuositatem, 11). In Zusammenarbeit mit den Eltern können die Medien und die Unterhaltungsindustrie in der schwierigen, aber hohe Erfüllung vermittelnden Aufgabe, Kinder zu erziehen, dadurch behilflich sein, dass sie aufbauende Beispiele für Leben und Liebe der Menschen darstellen (cf. Inter Mirifica, 11). Wie entmutigend und destruktiv ist es für uns alle, wenn das Gegenteil geschieht. Schmerzt nicht unser Herz in ganz besonderer Weise, wenn unsere jungen Menschen dem Einfluss von entwürdigenden oder falschen Ausdrucksformen von Liebe ausgesetzt sind, die die gottgegebene Würde jedes Menschen lächerlich machen und die Anliegen der Familien unterminieren?

4. Um zu einer konstruktiven Rolle und einer positiven Wahrnehmung der Medien in der Gesellschaft zu ermutigen, möchte ich erneut auf die Wichtigkeit von drei Schritten hinweisen, die mein verehrter Vorgänger 
Papst Johannes Paul II. beschrieben hat und die notwendig sind für den Dienst der Medien am Gemeinwohl: Erziehung, Teilhabe und Dialog (cf. Die schnelle Entwicklung, 11).

Erziehung zum verantwortungsvollen und kritischen Gebrauch der Medien hilft den Menschen, sie intelligent und angemessen zu nutzen. Die tiefe Wirkung auf den Sinn neuer Worte und Bilder, die besonders die elektronischen Medien so leicht in die Gesellschaft einführen, kann nicht hoch genug eingeschätzt werden. Eben weil zeitgenössische Medien die Kultur der Menschen prägen, müssen sie ihrerseits jeder Versuchung zur Manipulation, vor allem der Jugend, widerstehen und stattdessen dem Anliegen folgen, $z u$ erziehen und zu dienen. Auf diese Weise beschädigen sie nicht, sondern schützen das soziale Gewebe einer zivilen Gesellschaft, die des Menschen als einer Person würdig ist.

Teilhabe an den Medien entsteht aus ihrer Natur als einem Gut, das für alle Menschen bestimmt ist. Als eine öffentliche Dienstleistung erfordert soziale Kommunikation einen Geist der Zusammenarbeit und Mitverantwortung zusammen mit strenger Verantwortlichkeit im Gebrauch öffentlicher Ressourcen und der Wahrnehmung einer öffentlichen Treuhänderrolle (cf. Ethik in der Sozialen Kommunikation, 20) einschliesslich des Rückgriffs auf Regelungen und andere Massnahmen oder Strukturen, die dazu dienen, dieses Ziel zu verwirklichen.

Drittens, schliesslich, bieten die Förderung des Dialogs durch den Austausch im Lernen, der Ausdruck von Solidarität und der Einsatz für den Frieden eine grosse Gelegenheit für die Massenmedien, die erkannt und wahrgenommen werden muss. Auf diese Weise werden sie einflussreiche und geschätzte Ressourcen zur Entwicklung der Zivilisation der Liebe, wonach sich alle Völker sehnen.

Ich bin zuversichtlich, dass ernsthafte Bemühungen zur Realisierung dieser drei Schritte den Medien helfen werden, sich richtig zu entwickeln als ein Netzwerk von Kommunikation, Gemeinschaft und Zusammenarbeit sowie dabei Männern, Frauen und Kindern behilflich sind, sich der Würde des Menschen stärker bewusst zu werden, verantwortungsvoller und offener gegenüber anderen, besonders gegenüber den Bedürftigsten und schwächsten Mitgliedern der Gesellschaft (cf. Redemptor Hominis, 15; Ethik in der Sozialen Kommunikation, 4).

Abschliessend komme ich zurück auf die ermutigenden Worte des $\mathrm{Hl}$. Paulus: Christus ist unser Friede. In ihm sind wir eins (cf. Eph 2, 14). Lasst uns zusammen die trennenden Mauern der Feindschaft niederlegen und aufbauen die Gemeinschaft der Liebe nach dem Plan des Schöpfers, der uns durch se̊inen Sohn bekannt gemacht wurde!

Aus dem Vatikan, 24. Januar 2006, Fest des Hl. Franz von Sales

(C) Copyright 2006 - Libreria Editrice Vaticana 